\title{
Call for Papers: Special Issue on Bioelectricity for Cancer
}

\author{
Guest Editors: Mustafa Djamgoz, PhD and Annarosa Arcangeli, MD, PhD
}

Deadline for submission: June 1, 2019

\section{Special Issue Publication date: September 2019}

Cancer is a major killer of man in the modern world with one in two men and one in three women expected to be diagnosed with some kind of cancer during his/her lifetime. The statistics are expected to rise by more than $70 \%$ over the next two decades. Although great progress has been made in clinical management of cancer, many problems remain. These include uncertainties in diagnostics, limited effectiveness and undesirable side effects of major treatment modalities like chemotherapy, radiotherapy, and biological therapies, as well as cost. Thus, there is great unmet need in cancer management. Work aimed at understanding the bioelectrical properties of cancer cells and tissues in the last two decades has revealed a variety of novel targets, especially ion channels. It is likely, in fact, that cancer expresses as many ion channels as brain and these contribute to different components and stages of the dynamic process of cancer and response to therapy.

This special issue of Bioelectricity will consider articles from the full breadth of evidence: from original research papers and reviews, to perspectives, profiles, commentaries, and exemplary front matter. Both basic, especially molecular, and clinical aspects will be covered. Areas of specific interest include, but are not limited to:

- Ion channels

- Exchangers

- Pumps

- Field potentials

- Surface potentials

- Galvanotaxis

Contributions will receive prompt and thorough peer review. Please refer to our Instructions for Authors before submitting your manuscript for consideration. Authors may wish to vet ideas directly with the Guest Editors via e-mail before submitting (Mustafa Djamgoz, m.djamgoz@imperial.ac.uk; Annarosa Arcangeli, annarosa .arcangeli@unifi.it).

Bioelectricity is the only journal devoted explicitly to bioelectricity research. The field of bioelectricity, much like the field of biochemistry, is becoming a foundational component of the biological sciences. The scientists who pioneered modern studies of bioelectricity have created a solid footing for the imminent upsurge in exploration: Voltage, current, resistance, $\mathrm{pH}$, and other familiar ion-dependent phenomena, have all been found to be generated and maintained by cells in highly conserved, energydependent processes. Spearheaded by pioneering Editor-in-Chief, Dany Spencer Adams and Executive Editor Mike Levin, Bioelectricity will be published online and in print with open access options and rapid turnaround of submissions. 